Atherosclerosis

Elsevier Editorial System(tm) for

Manuscript Draft

Manuscript Number: ATH-D-18-00445R1

Title: High burden of recurrent cardiovascular events in heterozygous familial hypercholesterolemia: the French Familial Hypercholesterolemia Registry

Article Type: Research paper

Section/Category: Clinical \& Population Research

Keywords: familial hypercholesterolemia, registry, cardiovascular disease, cardiovascular recurrences, cardiovascular events

Corresponding Author: Dr. Sophie Béliard, MD, PhD

Corresponding Author's Institution: Aix Marseille Univ, INSERM, INRA, C2VN, Marseille, France

First Author: Sophie Béliard, MD, PhD

Order of Authors: Sophie Béliard, MD, PhD; Franck Boccara, MD, PhD; Bertrand Cariou, MD, PhD; Alain Carrié, MD, PhD; Xavier Collet, PhD; Michel Farnier, MD, PhD; Jean Ferrières, MD, PhD; Michel Krempf, MD, PhD; Noel Peretti, MD, PhD; Jean-Pierre Rabès, MD, PhD; Alexandre Vimont; Mathilde Varret, PhD; Sybil Charrières, MD, PhD; Eric Bruckert, MD, PhD

Abstract: Background and aims: Cardiovascular risk is high in heterozygous familial hypercholesterolemia (HeFH). The objective of this study was to describe recurrent cardiovascular events in selected patients with HeFH attending lipid clinics in France.

Methods: We included 781 patients with a clinical (Dutch Lipid Clinic Network score $\geq 6$ ) or genetic diagnosis of HeFH who had experienced a first cardiovascular event (myocardial infarction, percutaneous coronary intervention or coronary bypass, unstable angina, stroke, peripheral arterial revascularization or cardiovascular death) and were enrolled in the French Familial Hypercholesterolemia Registry (November 2015 to March 2018 ).

Results: The first cardiovascular event occurred at the mean age of 47 years (interquartile range 39-55) in a predominantly male population (72\%); 48\% of patients were on statin therapy. Overall, 37\% of patients had at least one recurrent cardiovascular event (mean of 1.8 events per patient), of which 32\% occurred in the 12 months after the index event; 55\% of events occurred $>3$ years after the first event. Mean LDL-C at the last clinic visit was $144 \pm 75 \mathrm{mg} / \mathrm{dL}$ (132 $169 \mathrm{mg} / \mathrm{dL}$ for patients on highpotency statin therapy and $223 \pm 85 \mathrm{mg} / \mathrm{dL}$ for untreated patients). Conclusions: The rate of recurrent cardiovascular events was high in French patients with HeFH in secondary prevention. The detection of $\mathrm{FH}$ in the childhood is crucial to prevent CV events at a young age by early initiating statin therapy. There is a clear urgent need to expand the actual very small target population which can be treated with PCSK9 inhibitor in France. 

One in two HeFH patients is not treated with statins at the time of the first CV even $37 \%$ of $\mathrm{HeFH}$ patients have $\mathrm{CV}$ recurrences after a first CV event

Nearly half of the $\mathrm{CV}$ recurrences occurred more than 3 years after the first event 


\section{High burden of recurrent cardiovascular events in heterozygous familial hypercholesterolemia: the French Familial Hypercholesterolemia Registry}

Short title: Cardiovascular recurrences in familial hypercholesterolemia

Sophie Béliard ${ }^{a, b, *}$, Franck Boccara ${ }^{c}$, Bertrand Cariou ${ }^{d}$, Alain Carrié $^{e}$, Xavier Collet $^{\dagger}$, Michel Farnier ${ }^{g}$, Jean Ferrières ${ }^{h}$, Michael Krempf ${ }^{d}$, Noël Peretti ${ }^{i}$, Jean-Pierre Rabès ${ }^{j}$, Mathilde Varret ${ }^{k}$, Alexandre Vimont ${ }^{1}$, Sybil Charrière ${ }^{m}$, Eric Bruckert ${ }^{n}$, French FH Registry group $\dagger$

${ }^{a}$ Aix Marseille Univ, INSERM, INRA, C2VN, Marseille, France

${ }^{\mathrm{b}}$ APHM, Department of Nutrition, Metabolic diseases, Endocrinology, La Conception Hospital, Marseille, France

${ }^{c}$ AP-HP, Hôpitaux de l'Est Parisien, Hôpital Saint-Antoine, Service de Cardiologie Faculty of Medicine, Sorbonne Université, National Institute of Health and Medical Research, INSERM, UMR_S 938, UPMC, Paris, France

'L'institut du thorax, Endocrinology department, CIC 1413 INSERM, CHU de Nantes, Nantes, France

e Sorbonne University, Inserm, Institute of Cardiometabolism and Nutrition (ICAN), UMR_S1166,

APHP, Department of Biochemistry, Obesity and Dyslipidemia Genetics Unit, Hôpital de la Pitié,

Paris, France

${ }^{\dagger}$ INSERM, UMR1048, Institute of Metabolic and Cardiovascular Diseases, University of Toulouse, Toulouse, France

g Point Medical and Department of Cardiology, CHU Dijon-Bourgogne, 21000 Dijon

${ }^{\text {h }}$ Department of Cardiology, INSERM UMR 1027, Toulouse University Hospital, Toulouse University School of Medicine, Toulouse, France

' Hospices Civils de Lyon, Department of Pediatric Nutrition, Bron, France

'Department of Biochemistry and Molecular Biology, Ambroise Paré Hospital, HUPIFO (APHP/UVSQUFR Sciences de la Santé Simone Veil), Boulogne-Billancourt, France

${ }^{k}$ LVTS, INSERM U1148, Paris Diderot University, Paris 7, France

'PHExpertise, Paris, France 
${ }^{\mathrm{m}}$ Hospices Civils de Lyon, Endocrinology department, Bron, Lyon1 University, France

${ }^{n}$ Department of Endocrinology and Cardiovascular Disease Prevention, Institute of Cardio

Metabolism And Nutrition (ICAN), La Pitié-Salpêtrière Hospital, AP-HP, Paris, France

* Corresponding author. APHM, Department of Nutrition, Metabolic diseases, Endocrinology, La Conception Hospital,

147 boulevard Baille, 13005 Marseille, France.

E-mail address: sophie.beliard@ap-hm.fr (S. Beliard).

Tel: $+33-491-383-650$

Fax: +33-491-384-132

Key words: familial hypercholesterolemia, registry, cardiovascular disease, cardiovascular recurrences, cardiovascular events

tFrench FH Registry group: D. Angoulvant, S. Béliard, P. Benlian, C. Boileau, F. Boccara, E. Bruckert, B. Cariou, V. Carreau, A. Carrié, S. Charrière, M. Di Filippo, PH. Ducluzeau, S. Dulong, V. Durlach, M. Farnier, E Ferrari, J. Ferrières, A. Gallo, JP. Girardet, R. Hankard, M. Krempf, JD. Lalau, B. Lefort, J. Lemale, P. Moulin, F. Paillard, N. Peretti, A. Pradignac, Y. Pucheu, JP. Rabès, S. Saheb,
A Sultan, P. Tounian, R. Valéro, M. Varret,
B. Vergès, C Yelnik,
O. Ziegler. 
Keywords:

Familial hypercholesterolemia

Registry

Cardiovascular disease

Cardiovascular recurrences

Cardiovascular events 


\section{Introduction}

Familial hypercholesterolemia $(\mathrm{FH})$ is an autosomal dominant hereditary disease caused by mutations in genes involved in the catabolism of low-density lipoprotein cholesterol (LDL-C): LDLR, $A P O B$ and PCSK9 [1,2]. $\mathrm{FH}$ is one of the most common genetic diseases, with an estimated prevalence of 1 in 250 for heterozygous $\mathrm{FH}(\mathrm{HeFH})$ and 1 in 300,000 for homozygous $\mathrm{FH}$ [3-5]. People with FH have very high circulating levels of LDL-C from birth, leading to a lifelong exposure of the arteries to elevated levels of cholesterol and a high cardiovascular risk [6,7]. Young adults with FH have an up to 13-fold higher risk of myocardial infarction compared to similar young adults without the condition [3,4]. As placebo-controlled randomized trials with cardiovascular endpoints are unethical in $\mathrm{FH}$, the reduction in cardiovascular risk with lipid-lowering treatment has to be extrapolated from trials in the general population or from observational studies [8-10].

The current burden of cardiovascular disease in patients with HeFH in France is unknown. We sought to evaluate the typical features of cardiovascular disease (mean age at the time of the event, type and number of events, use of lipid-lowering therapies) in HeFH patients in secondary prevention in the French Familial Hypercholesterolemia Registry.

\section{Patients and Methods}

\subsection{Settings}

In 2015, the New French Society of Atherosclerosis established a national multicenter registry to identify patients with $\mathrm{FH}$ in France, with the objective of assessing screening practices, treatments, and clinical and patient-reported outcomes. Eligible patients were those who visited a participating lipid clinic and were diagnosed, either clinically (Dutch Lipid Clinic Network score $\geq 6$ ) or genetically, with homozygous or heterozygous $\mathrm{FH}$.

Sixteen sites contributed data to the registry, as of March 2018. Adult patients received an information leaflet about the registry before being evaluated for inclusion. Informed consent from both parents was required in the case of minors. The cohort was declared to the ANSM (the French National Agency for Medicines safety) and received a declarant number [unique number identifying a particular research protocol, issued by the ANSM in France]: 2014-A01549-38. The protocol of this study was assessed by two different committees: French advisory committee on the processing of information for medical research (CCTIRS) and the National Commission for computer technology and 
freedom (CNIL) respectively in May and November 2015. This research is conducted in accordance with good clinical practices.

Clinical and laboratory data from the patients' medical records were obtained during routine clinic visits and were entered into the registry database by trained research staff. To obtain data from first contact, retrospective data extraction from the patients' medical records was done for patients who were already being treated at the site.

Sociodemographic, clinical and biological data (age, height, weight, blood pressure, smoking status, cardiovascular events, use of lipid-lowering drugs) were collected, along with family history (hypercholesterolemia or any cardiovascular disease), standard laboratory results (total cholesterol, LDL-C) and cardiovascular imaging data. Where available, genetic data (presence of known FHrelated mutations) were collected. Information on cardiovascular history included the date of diagnosis and the type of event. Cardiovascular events included coronary heart disease (acute coronary syndromes, encompassing myocardial infarction and unstable angina, defined according to the European Society of Cardiology/American College of Cardiology [11], percutaneous coronary intervention or coronary artery bypass graft), stroke or transient ischemic attack, peripheral artery disease (defined as carotid endarterectomy, carotid angioplasty, peripheral artery angioplasty or bypass), resuscitated sudden death and cardiovascular death.

\subsection{Patients and Outcomes}

The present analysis involved patients with either a Dutch Lipid Clinic Network score $\geq 6$ or an FHcausing mutation, plus a history of at least one cardiovascular event. Cardiovascular event and recurrences of cardiovascular events were defined as: coronary heart disease (myocardial infarction, coronary angioplasty or bypass and unstable angina), stroke, transient ischemic attack, peripheral artery disease (carotid endarterectomy, carotid angioplasty, and peripheral arterial bypass), resuscitated sudden death or cardiovascular death. A recurrent cardiovascular event was defined as a second cardiovascular event, regardless of the type of the first or the second event. Coronary revascularization (i.e. percutaneous coronary intervention or coronary artery bypass graft) and myocardial infarction occurring $<30$ days after the index event were not considered as recurrences.

Overall follow-up duration was defined as the time from the first cardiovascular event to the last available visit at the lipid clinic. The length of time between the first and the last available visit was also 
assessed. Exclusion criteria were a diagnosis of homozygous $\mathrm{FH}$ and the occurrence of minor cardiovascular events such as silent ischemia and stable angina when not followed by coronary revascularization.

\subsection{Statistical analysis}

Data are presented as frequency and percentage for categorical variables and as mean (standard deviation [SD]) or median with interquartile range (IQR) for continuous variables. A descriptive analysis was performed for cardiovascular event history. The characteristics of patients with and without at least one recurrence were compared using Student's $t$ test or the Wilcoxon ranksum test for continuous variables, and the chi-square test or Fisher's exact test for categorical variables. $P$ values were associated with a significance level of 0.05 for all tests. All analyses were performed with SAS version 9.4 software (SAS Institute Inc., Cary, NC, USA).

\section{Results}

\subsection{Patient characteristics}

Between November 2015 and March 2018, 4682 patients were enrolled in the registry, of which $781(16.7 \%)$ met the inclusion criteria. The characteristics of the population (at the last clinic visit) are detailed in Table 1. Median follow-up was 7 (IQR: 2-17) years from the time of the first cardiovascular event until the last available clinic visit, totaling 5779 patient-years of follow-up. The population was predominantly male $(72 \%)$ with a mean age of 60 years, and $64 \%$ had a family history of any cardiovascular event. Over half $(55 \%)$ of the patients had a history of smoking, $36 \%$ had hypertension and $13 \%$ had type 2 diabetes. Data on maximum lifetime cholesterol and lipoprotein(a) were available for $535(69 \%)$ and $414(53 \%)$ patients, respectively; mean (SD) lifetime cholesterol was 420 (19) $\mathrm{mg} / \mathrm{dL}$ and lipoprotein(a) was 55 (56) $\mathrm{mg} / \mathrm{dL}$. Overall, $75 \%$ patients underwent genetic testing, of which $437(75 \%)$ had a mutation, with some patients having mutations in more than one gene. Median age at the first visit to a lipid clinic was 51 (IQR: 41-60) years. 


\subsection{Index and recurrent cardiovascular events}

Information on history of cardiovascular events, collected at the last clinic visit, is shown in Table 2. Median age at the first cardiovascular event was 47 (IQR 39-55) years. The most frequently observed first events were coronary revascularization procedure (64\%) and myocardial infarction $(27 \%)$.

Overall, 511 recurrences were observed, yielding an average incidence rate of 9 per 100 patientyears after the first cardiovascular event. During follow-up, $37 \%$ of patients had at least one recurrence, $14 \%$ had two or more recurrences and $7 \%$ had three or more recurrences. The most frequent recurrences were coronary revascularization (65\%) and peripheral artery disease (15\%). The median time between the first and the second event was 4 years (49 months), with a median duration of 3 years (38 months) between recurrences. For $3 \%$ of patients, the recurrence occurred during the first 30 days after the first event and $29 \%$ in the first year. Approximately half of the patients had a second cardiovascular event $>3$ years after the first event. Median age at the time of the second event was 49 years (IQR: 42-57). A Kaplan-Meier curve showing freedom from recurrent cardiovascular events is shown in Figure 1.

\subsection{Patients with recurrent cardiovascular events}

Compared with patients who did not have a recurrent cardiovascular event, those with a recurrent event were more likely to be male ( $81 \%$ vs $67 \%, p<0.0001)$ and to be older (62 vs 59 years, $p<$ 0.0001) (Table 3). No differences were found in terms of genetic mutations, lipoprotein(a) levels, and maximum lifetime total cholesterol.

In patients with a recurrent event, the first cardiovascular event had occurred 7 years earlier $(p<$ 0.0001), at a median age of 42 years (IQR 37 - 51) (Table 3). The type of first cardiovascular event was more often myocardial infarction ( $28 \%$ vs $18 \%$, respectively) and less often stroke or TIA ( $6 \%$ vs $13 \%$, respectively) in patients with at least one recurrence $(p=0.0070)$.

\subsection{Treatment patterns in patients with or without cardiovascular recurrences}

Median duration of statin therapy was 19 years (IQR: 10-27), starting at a median age of 41 years (IQR: 33-50); $48 \%$ of patients were treated with statin at the time of the first cardiovascular event (Table 4). Patients with at least one recurrence were less likely to be on statin treatment at the time of 
the first event $(39 \%$ vs $53 \%, p=0.0084)$. The mean length of exposure to statins before the first event was shorter in patients with a cardiovascular recurrence (6.2 years vs 8.6 years, $p=0.0191$ ).

At the time of the last clinic visit, $83 \%$ of patients were receiving statin therapy (alone or in combination with other lipid-lowering therapy); $58 \%$ were on a high-potency statin, as defined by Law et al. [12], and $57 \%$ were on statin plus ezetimibe (Table 4). No difference in treatments were found between patients with or without cardiovascular recurrences $(p=0.0690)$.

At the last clinic visit, mean LDL-C was $144 \pm 75 \mathrm{mg} / \mathrm{dL}$ for all patients $(\mathrm{n}=714), 132 \pm 69 \mathrm{mg} / \mathrm{dL}$ for patients on high-potency statin therapy $(n=456)$ and $223 \pm 85 \mathrm{mg} / \mathrm{dL}$ for untreated patients $(n=58)$. When reported $(52 \%, 44 / 84)$, reasons for not taking statin therapy were muscle symptoms $(44 \%$, $37 / 84)$, patient refusal $(4 \%, 3 / 84)$ and hepatic intolerance $(4 \%, 3 / 84)$.

\section{Discussion}

The results from this contemporary French multicenter registry demonstrate the high burden of cardiovascular disease in $\mathrm{HeFH}$. One in two patients was not receiving statin treatment at the time of their first cardiovascular event, which occurred at a mean age of 47 years, and only $58 \%$ were on a high-potency statin. Recurrent events occurred in 37\% of patients, with almost two events (first and recurrent) per patient.

\subsection{Statins in primary prevention}

In $\mathrm{FH}$, initiation of statin treatment early in life decreases long-term exposure of the arteries to high cholesterol levels and is highly beneficial in the prevention of cardiovascular events [13]. US and European guidelines recommend starting statins before the age of 10 years in $\mathrm{FH}[1,2,14]$. French pediatric and atherosclerosis societies also promote the initiation of statins during childhood in $\mathrm{FH}$ $[15,16]$. In our study, approximately $40 \%$ of patients started statin therapy in primary prevention, suggesting that the hypercholesterolemic status of the remaining patients before the first event was either unknown or ignored by patients and/or their general practitioners. Furthermore, the mean age at which statin treatment was started was 41 years, by which time these patients have been exposed to elevated cholesterol levels for many years, placing them at high cardiovascular risk $[1,15,16]$.

\subsection{Recurrent events}


Recurrent cardiovascular events are associated with poor quality of life as well as high costs due to hospitalization and physician visits [17]. In a Swedish national registry of patients post myocardial infarction, the probability of a recurrent event (defined as non-fatal myocardial infarction, non-fatal stroke or cardiovascular death) in patients aged $<60$ years within 12 months of the index event was $6.5 \%$, and the rate remained relatively stable to 3 years [18]. In the UK cohort of the GRACE UKBelgium registry, published in 2010, recurrent myocardial infarction occurred in $15.5 \%$ of patients with an acute coronary syndrome up to 5 years after the first cardiovascular event [19]. Rapsomaniki et al. reported a $9.7 \%$ incidence of recurrent cardiovascular events (myocardial infarction or coronary heart disease death) at 5 years in a population with stable coronary artery disease [20]. The recurrence rates in these studies, albeit in different populations and with different endpoints, are markedly lower than in our registry of HeFH (9 per 100 patient-years).

Contemporary data on recurrent cardiovascular events in HeFH are scarce. Recently, a Swiss study reported a high risk of recurrent coronary events (odds ratio of 2-3.5 versus non- $\mathrm{FH}$, depending on the criteria used for the clinical diagnosis of $\mathrm{FH}$ ) in $\mathrm{HeFH}$ patients after an acute coronary syndrome [21]. In an analysis using data from two Danish registries, Rerup et al. demonstrated that patients with a possible diagnosis of $\mathrm{FH}$ (based on the Dutch Lipid Clinic Network criteria) had a higher risk of recurrent myocardial infarction than those without $\mathrm{FH}$ (16\% vs $11 \%$, respectively, over a period of 3.3 years) [22]. In 2018, Galema-Boers et al. reported a $30 \%$ rate of subsequent cardiovascular events in a Dutch cohort of 102 patients with HeFH (diagnosed using the same criteria as in our study), despite maximum-tolerated lipid-lowering therapy [23].

The design of our study may in part explain the high rate of recurrent cardiovascular events. First, data were collected over a period exceeding 7 years, versus 1 year in the Swiss study [21] and 3.3 years in the Danish study [22]. Second, $75 \%$ of the patients were diagnosed using genetic testing; among those diagnosed clinically, a Dutch Lipid Clinic Network score of $\geq 6$ was used (indicating a probable or definite diagnosis). Third, our population may be biased towards patients with moresevere $\mathrm{FH}$, as it was restricted to those referred to lipid clinics.

\subsection{Cardiovascular events}

The majority of first and subsequent cardiovascular events (approximately $80 \%$ ) were coronary in origin, with peripheral artery disease and ischemic stroke occurring in roughly equal proportions 
(approximately $10 \%$ each). In a Spanish study, Mata et al. described a higher proportion of coronary heart disease (93.4\%) in HeFH patients with prior cardiovascular disease [24]. Few data are available on cardiovascular events other than coronary events in FH studies [3]. Most recurrences (65\%) were new revascularization procedures, which is in line with other studies in distinct populations. For example, $58 \%$ of recurrent events were coronary revascularization in the IMPROVE-IT trial in patients after an acute coronary syndrome [25].

\subsection{Cardiovascular risk factors}

More than half of our patients were current or former smokers. In a previous study in 734 patients with $\mathrm{HeFH}$ (with or without cardiovascular events), $75 \%$ had at least one additional risk factor, of which $31 \%$ was tobacco smoking [26]. These results emphasize the importance of identifying ways in which to encourage smoking cessation in these high cardiovascular risk patients.

The results of our study confirm that patients with $\mathrm{FH}$ in secondary prevention are undertreated with lipid-lowering therapy and are not achieving the recommended LDL-C target of $70 \mathrm{mg} / \mathrm{dL}$ [7]. Despite the very high cardiovascular risk of these patients, the mean LDL-C value was $144 \pm 77 \mathrm{mg} / \mathrm{dL}$. These findings are in concert with our previous work, in which LDL-C levels remained elevated despite maximal therapy with a high-potency statin plus a non-statin lipid-lowering drug (mainly ezetimibe) [27], and show that even when managed by lipid specialists, the majority of patients with HeFH have LDL-C levels that are above the recommended target. Whereas the high levels of LDL-C in HeFH reflect the disease itself, statin intolerance - primarily manifesting as muscle symptoms - can also lead to inability to take statins or to tolerate only low doses. In France, the PCSK9 inhibitors have been reimbursed since February 2018 for two conditions: alirocumab for $\mathrm{HeFH}$ requiring lipoprotein apheresis; and evolocumab for homozygous FH. In France, the criteria for apheresis are LDL-C >200 $\mathrm{mg} / \mathrm{dL}$ on maximum-tolerated oral lipid-lowering therapy in secondary prevention, and LDL-C >300 $\mathrm{mg} / \mathrm{dL}$ on maximum-tolerated oral lipid-lowering therapy in primary prevention.

\subsection{Limitations}

Whereas registries provide data from the spectrum of patients treated in everyday clinical practice, several limitations must be acknowledged. First, as we collected data from patients referred to lipid centers, our population may be biased towards more severe patients with HeFH. However, the 
prevalence of comorbidities are consistent with data from other FH registries. Second, we could not verify the reason for recurrent revascularization, whether ischemia-driven, urgent or due to the presence of silent ischemia. Third, it is important to note that data regarding the initiation of statin therapy before the first event were absent for almost half of the patients, so the results on exposure on statins may not be representative of the entire population.

\subsection{Conclusions}

The rate of recurrent cardiovascular events was high in this retrospective, contemporary French study of HeFH. A substantial proportion of these high-risk patients were undertreated with lipidlowering therapies and were not achieving the recommended LDL-C target. Detection of FH in the childhood is crucial to prevent cardiovascular events at a young age, through early initiation of statin therapy. There is also clear and urgent need to ensure that non-statin lipid-lowering therapies, such as the PCSK9 inhibitors, are considered in patients with $\mathrm{HeFH}$ who are unable to achieve the recommended LDL-C target with their current therapy alone.

Author's contributions: SB and EB are the principal investigators of the French FH registry; they contributed to the design and the setting of the registry. SB and EB wrote the manuscript. AV performed the statistical analyses. FB, BC, AC, JF, MF, MK, MV, SC, NP and JPR are coinvestigators, and made critical revisions to the manuscript. SB and EB are the guarantors of this work and, as such, had full access to all of the data and take responsibility for the integrity of the data.

\section{Conflicts of interest}

$\mathrm{SB}, \mathrm{EB}, \mathrm{BC}, \mathrm{FB}, \mathrm{AC}, \mathrm{JF}, \mathrm{MF}, \mathrm{MK}, \mathrm{MV}, \mathrm{SC}, \mathrm{NP}$ and JPR have received honoraria from Sanofi and Amgen for board, conferences, clinical trials, or congresses. AV has no conflict of interest to declare.

\section{Acknowledgments}

The authors thank Amgen and Sanofi laboratories and the NSFA for helping to sponsor the French Familial Hypercholesterolemia Registry. We thank Dr Leleu (PH Expertise) for help with the statistical analyses. This work was supported by the French national project CHOPIN (CHolesterol Personalized Innovation), with a grant from the Agence Nationale de la Recherche (ANR-16-RHUS-0007), 
coordinated by the CHU of Nantes. Editorial support was provided by Sophie K. Rushton-Smith, PhD (MedLink Healthcare Communications, London), and was funded by French National Society of Atherosclerosis.

\section{References}

[1] Nordestgaard BG, Chapman MJ, Humphries SE, et al. Familial hypercholesterolaemia is underdiagnosed and undertreated in the general population: guidance for clinicians to prevent coronary heart disease: consensus statement of the European Atherosclerosis Society. Eur Heart J 2013; 34:3478-3490a.

[2] Cuchel M, Bruckert E, Ginsberg HN, et al. Homozygous familial hypercholesterolaemia: new insights and guidance for clinicians to improve detection and clinical management. A position paper from the Consensus Panel on Familial Hypercholesterolaemia of the European Atherosclerosis Society. Eur Heart J 2014; 35:2146-2157.

[3] Benn M, Watts GF, Tybjaerg-Hansen A, Nordestgaard BG. Familial hypercholesterolemia in the danish general population: prevalence, coronary artery disease, and cholesterol-lowering medication. J Clin Endocrinol Metab 2012; 97:3956-3964.

[4] Do R, Stitziel NO, Won HH, et al. Exome sequencing identifies rare LDLR and APOA5 alleles conferring risk for myocardial infarction. Nature 2015; 518:102-106.

[5] Sjouke B, Kusters DM, Kindt I, et al. Homozygous autosomal dominant hypercholesterolaemia in the Netherlands: prevalence, genotype-phenotype relationship, and clinical outcome. Eur Heart J 2015; 36:560-565.

[6] Reiner Z, Catapano AL, De Backer G, et al. ESC/EAS Guidelines for the management of dyslipidaemias: the Task Force for the management of dyslipidaemias of the European Society of Cardiology (ESC) and the European Atherosclerosis Society (EAS). Eur Heart J $2011 ; 32: 1769-1818$.

[7] Catapano AL, Graham I, De Backer G, et al. 2016 ESC/EAS Guidelines for the Management of Dyslipidaemias: The Task Force for the Management of Dyslipidaemias of the European Society of Cardiology (ESC) and European Atherosclerosis Society (EAS) Developed with the special contribution of the European Assocciation for Cardiovascular Prevention \& Rehabilitation (EACPR). Atherosclerosis 2016; 253:281-344. 
[8] Krogh HW, Mundal L, Holven KB, Retterstol K. Patients with familial hypercholesterolaemia are characterized by presence of cardiovascular disease at the time of death. Eur Heart $\mathrm{J}$ 2016; 37:1398-1405.

[9] Mortality in treated heterozygous familial hypercholesterolaemia: implications for clinical management. Scientific Steering Committee on behalf of the Simon Broome Register Group. Atherosclerosis 1999; 142:105-112.

[10] Mundal L, Sarancic M, Ose L, et al. Mortality among patients with familial hypercholesterolemia: a registry-based study in Norway, 1992-2010. J Am Heart Assoc 2014; 3:e001236.

[11] Alpert JS, Thygesen K, Antman E, Bassand JP. Myocardial infarction redefined--a consensus document of The Joint European Society of Cardiology/American College of Cardiology Committee for the redefinition of myocardial infarction. J Am Coll Cardiol 2000; 36:959-969.

[12] Law MR, Wald NJ, Rudnicka AR. Quantifying effect of statins on low density lipoprotein cholesterol, ischaemic heart disease, and stroke: systematic review and meta-analysis. BMJ 2003; 326:1423.

[13] Versmissen J, Oosterveer DM, Yazdanpanah M, et al. Efficacy of statins in familial hypercholesterolaemia: a long term cohort study. BMJ 2008; 337:a2423.

[14] Goldberg AC, Hopkins PN, Toth PP, et al. Familial hypercholesterolemia: screening, diagnosis and management of pediatric and adult patients: clinical guidance from the National Lipid Association Expert Panel on Familial Hypercholesterolemia. J Clin Lipidol 2011; 5:S1-8.

[15] Besseling J, Kindt I, Hof M, Kastelein JJ, Hutten BA, Hovingh GK. Severe heterozygous familial hypercholesterolemia and risk for cardiovascular disease: a study of a cohort of 14,000 mutation carriers. Atherosclerosis 2014; 233:219-223.

[16] Santos RD, Gidding SS, Hegele RA, et al. Defining severe familial hypercholesterolaemia and the implications for clinical management: a consensus statement from the International Atherosclerosis Society Severe Familial Hypercholesterolemia Panel. Lancet Diabetes Endocrinol 2016.

[17] Spertus JA, Radford MJ, Every NR, Ellerbeck EF, Peterson ED, Krumholz HM. Challenges and opportunities in quantifying the quality of care for acute myocardial infarction: summary from the Acute Myocardial Infarction Working Group of the American Heart 
Association/American College of Cardiology First Scientific Forum on Quality of Care and Outcomes Research in Cardiovascular Disease and Stroke. Circulation 2003; 107:1681-1691.

[18] Jernberg T, Hasvold P, Henriksson M, Hjelm H, Thuresson M, Janzon M. Cardiovascular risk in post-myocardial infarction patients: nationwide real world data demonstrate the importance of a long-term perspective. Eur Heart J 2015; 36:1163-1170.

[19] Fox KA, Carruthers KF, Dunbar DR, et al. Underestimated and under-recognized: the late consequences of acute coronary syndrome (GRACE UK-Belgian Study). Eur Heart J 2010; 31:2755-2764.

[20] Rapsomaniki E, Shah A, Perel P, et al. Prognostic models for stable coronary artery disease based on electronic health record cohort of 102023 patients. Eur Heart J 2014; 35:844-852.

[21] Nanchen D, Gencer B, Muller O, et al. Prognosis of Patients With Familial Hypercholesterolemia After Acute Coronary Syndromes. Circulation 2016; 134:698-709.

[22] Rerup SA, Bang LE, Mogensen UM, et al. The prevalence and prognostic importance of possible familial hypercholesterolemia in patients with myocardial infarction. Am Heart J 2016; $181: 35-42$.

[23] Galema-Boers AM, Lenzen MJ, Engelkes SR, Sijbrands EJ, Roeters van Lennep JE. Cardiovascular risk in patients with familial hypercholesterolemia using optimal lipid-lowering therapy. J Clin Lipidol 2018; 12:409-416.

[24] Mata N, Alonso R, Badimon L, et al. Clinical characteristics and evaluation of LDL-cholesterol treatment of the Spanish Familial Hypercholesterolemia Longitudinal Cohort Study (SAFEHEART). Lipids Health Dis 2011; 10:94.

[25] Murphy SA, Cannon CP, Blazing MA, et al. Reduction in Total Cardiovascular Events With Ezetimibe/Simvastatin Post-Acute Coronary Syndrome: The IMPROVE-IT Trial. J Am Coll Cardiol 2016; 67:353-361.

[26] Beliard S, Millier A, Carreau V, et al. The very high cardiovascular risk in heterozygous familial hypercholesterolemia: Analysis of 734 French patients. J Clin Lipidol 2016; 10:1129-1136 e1123.

[27] Beliard S, Carreau V, Carrie A, et al. Improvement in LDL-cholesterol levels of patients with familial hypercholesterolemia: can we do better? Analysis of results obtained during the past two decades in 1669 French subjects. Atherosclerosis 2014; 234:136-141. 
Fig. 1. Kaplan-Meier curve of freedom from recurrent cardiovascular event.

Coronary heart disease recurrences were censured when they were $<30$ days after a first coronary event. The shading shows $95 \%$ Hall-Wellner confidence interval.

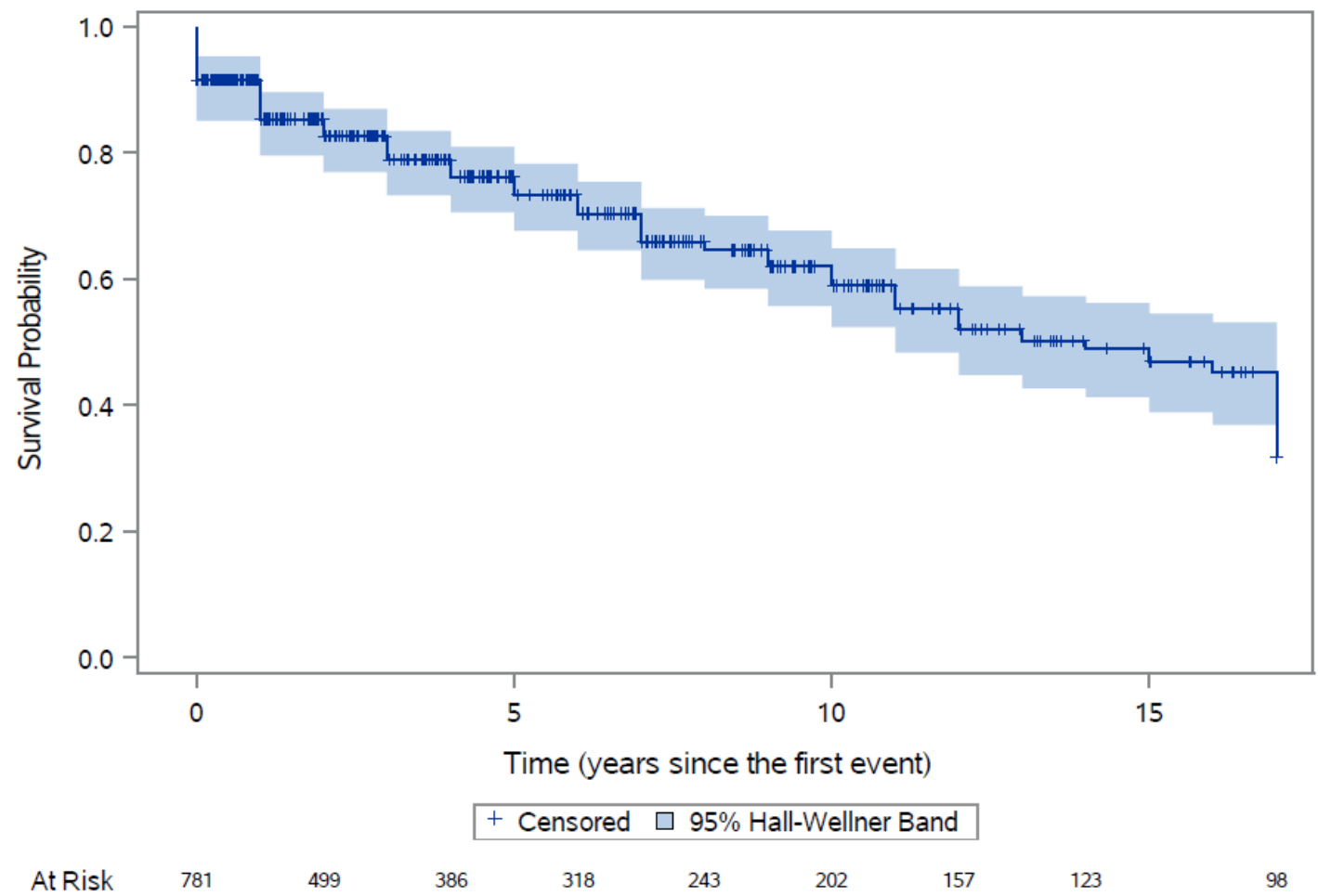




\section{REBUTTAL LETTER TO THE EDITOR AND THE REVIEWERS:}

Dear Professors von Eckardstein and Ray,

Thank you for reviewing our manuscript, entitled "High burden of recurrent cardiovascular events in heterozygous familial hypercholesterolemia: the French Familial Hypercholesterolemia Registry" (Ms. No. ATH-D-18-00445).

We again thank the reviewers for their overall positive comments and the pertinent points raised. Our responses to these comments are provided below, and the changes are marked in the manuscript.

We believe that the manuscript has benefited significantly from the reviewers' critiques, and hope that you will now find it acceptable for publication in your journal.

Yours sincerely,

Dr SOPHIE BELIARD, MD, PhD

Marseille, the 15 June 2018,

Reviewers' comments:

\section{Reviewer \#1:}

The manuscript entitled "High burden of recurrent cardiovascular events in Familial Hypercholesterolemia: data from the French FH Registry" has been evaluated and got the following comments. First of all, your work is interesting,and needs to be published. However, I found many flaws when revising the manuscript, and I think you should consider re-writing and re-submitting the article. One example is that you use $\mathrm{mg} / \mathrm{dL}$ to describe lipid values $(1,5$ $\mathrm{mg} / \mathrm{dL}, 0,70 \mathrm{mg} / \mathrm{dL}$ ), when these values should be in $\mathrm{g} / \mathrm{L}$. In the Tables the values are correctly presented, but not in the text. Indeed, we usually use lipid values in $\mathrm{mg} / \mathrm{dL}$ or in $\mathrm{mmol} / \mathrm{L}$. But, $\mathrm{OK}$, they are in $\mathrm{g} / \mathrm{L}$, but stated as $\mathrm{mg} / \mathrm{dL}$. That gives a totally different picture of your results.

Response: We thank the reviewer for his/her very positive and encouraging assessment of our manuscript and the pertinent points raised. First, we sincerely apologize for the errors in the units for the lipid values, which have now been corrected and presented in $\mathrm{mg} / \mathrm{dL}$. 
It would be nice to have a cumulative event rate curve (Kaplan-Meier) illustrating your recurrent events.

Response: As suggested, we have included a Kaplan-Meier curve (Figure 1).

Figure 1: Kaplan-Meier curve of freedom from recurrent cardiovascular event.

Coronary heart disease recurrences were censured when they were $<30$ days after a first coronary event. The shading shows $95 \%$ Hall-Wellner confidence interval.

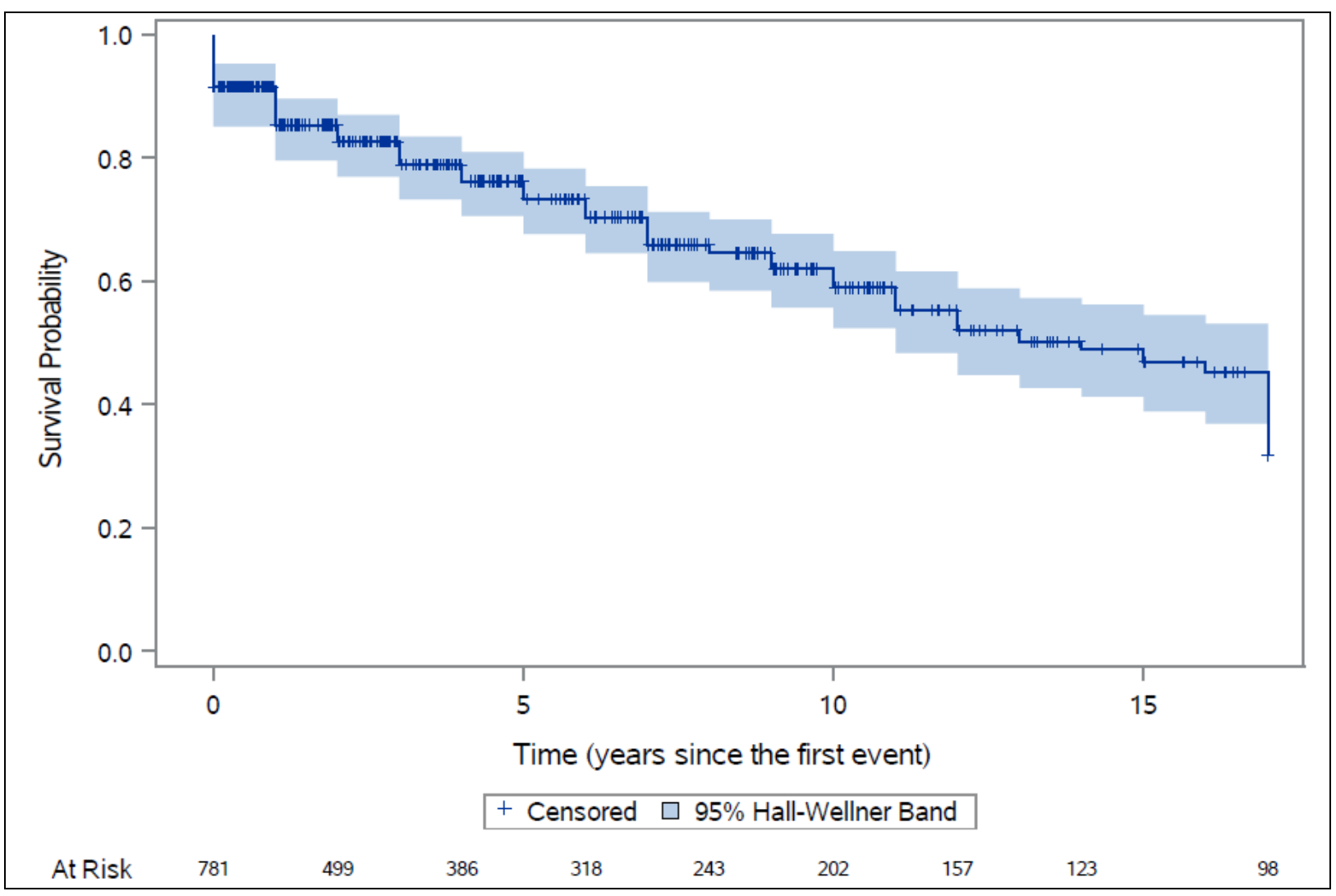

The English language must be carefully reviewed by a native English.

Response: As suggested, a native English speaker has reviewed the manuscript and corrected the language.

\section{Reviewer \#2:}

The study is very well designed, analyzed and presented. However, the main finding is the recurrence of $\mathrm{CV}$ events in heFH patients, which has been reported before in several studies and populations. The observation of increased CV event recurrences in French heFH is very important for French clinicians in order to modify the therapeutic approaches for this disease (e.g. expand the group of patients that are eligible for PCSK9 prescription). Response: We thank the reviewer for his/her very positive and encouraging assessment of our manuscript.

The high CV risk of the HeFH population is indeed well known, and has been studied in many cohorts (Benn et al. JCEM 2012, Do et al. Nature 2015, Mundal et al. J Am Heart Assoc 2014, Wallas Krogh et al. Eur Heart J 2015). However, we have found only 3 recent studies in the literature that present the 
recurrence of $\mathrm{CV}$ events in this specific population of patients with $\mathrm{HeFH}$. We discuss this point on page 9:

"Contemporary data on recurrent cardiovascular events in HeFH are scarce. Recently, a Swiss study reported a high risk of recurrent coronary events (odds ratio of 2-3.5 versus non-FH, depending on the criteria used for the clinical diagnosis of $\mathrm{FH}$ ) in HeFH patients after an acute coronary syndrome [20]. In an analysis using data from two Danish registries, Rerup et al. demonstrated that patients with a possible diagnosis of FH (based on the Dutch Lipid Clinic Network criteria) had a higher risk of recurrent myocardial infarction than those without $\mathrm{FH}$ (16\% vs $11 \%$, respectively, over a period of 3.3 years) [21]. In 2018, Galema-Boers et al. reported a 30\% rate of subsequent cardiovascular events in a Dutch cohort of 102 patients with HeFH (diagnosed using the same criteria as in our study), despite maximum-tolerated lipid-lowering therapy [22]."

The results of the study though do not add substantially new knowledge for $\mathrm{CV}$ events and $\mathrm{FH}$ to the international scientific community

Response: The strengths of this national study are the multicenter design (16 clinical reference centers for FH diagnosis and care) and the long follow-up (median 7.4 years). We believe that our article offers new insights to the scientific community, with the longest follow-up of FH patients with CVD. Other studies had shorter follow-up periods (1 year for the Swiss cohort, Nanchen D et al. Circulation 2016; 134:698-709) and 3.3 years for Rerup SA et al (Am Heart J 2016; 181:35-42); duration of follow-up was not mentioned in the paper by Galema-Boers et al ( $\mathrm{J}$ Clin Lipidol 2018; 12:409-416).

We observed that $68.3 \%$ of the recurrences occurred after the first year, emphazing the need for longterm cardiovascular follow-up in this population. Finally, as you acknowledge, we hope that our results will provide support to extend the criteria for reimbursement of PCSK9 monoclonal antibodies in patients with severe $\mathrm{FH}$ in France.

\section{Reviewer \#3:}

The absence for ethical reasons of clinical trials in familial hypercholesterolemia (FH) makes the information derived from registers have a special value. The French registry recruits subjects with FH from 18 different units of France and in this work presents information on recurrences of events. This issue is especially important among other things because the lipidlowering treatment in these and other subjects depends on the baseline CVD risk, considering the price of some drugs. The work provides new information of clinical interest that, nevertheless, has to clarify several aspects.

Response: We thank the reviewer for his/her positive and encouraging review of our manuscript.

-It is not clear in the article the temporal distribution of information. The data in Table 1 should be better explained. It is difficult to know if the information refer to the diagnosis of $\mathrm{FH}$, to the 
first visit in the lipid unit, at the time of inclusion in the register, at the time of the event or during the follow-up.

-The same applies to the information in Table 2. Are the data at the time of recurrence, during follow-up, or the last visit?

Response: We have clarified the information presented in Tables 1 and 2. These data were collected during the patients' last visits to the lipid clinic.

-The definition of event is not very solid. The high number of peripheral arterial disease is striking. What criteria were used for its diagnosis?

Response: We have now included the definition for acute coronary syndrome in the manuscript (Alpert JS, et al. Myocardial infarction redefined--a consensus document of The Joint European Society of Cardiology/American College of Cardiology Committee for the redefinition of myocardial infarction. J Am Coll Cardiol 2000; 36:959-969). We have also modified our definition of peripheral artery disease (PAD), so that it includes only PAD that required intervention, such as carotid endarterectomy, carotid angioplasty, and peripheral arterial bypass. Therefore, the number of PAD events decreased from 99 to 76 . All these changes are in red in the manuscript (page 5).

-The inclusion of valvulopathy is very debatable and it should be defined what criteria was used. Any valve of any etiology?

Response: We agree with the reviewer: valvulopathy may not be due to atherosclerosis and should not be included in the analysis. We have therefore excluded the data from patients who had valve replacement or aortic intervention. Twenty events were excluded from the analysis, leading to 781 events instead of 801.

- $40 \%$ of the events occur after the first year of follow-up, and a large part of them in the first month.

Response: We have recalculated the number of events, excluding coronary events (recurrent $\mathrm{Ml}$, recurrent coronary revascularization) that occurred within 30 days after the index event. All these changes are in red in the manuscript (page 5).

This type of events is complicated to attribute to recurrence since they are possibly intimately related to the index event.

Response: We have censured coronary events that occurred less than 30 days after the index event, to exclude any that could be attributed to the index event.

- In relation to the previous comment, $61 \%$ of the recurrences are coronary revascularizations, when they were $32 \%$ of the first event. These differences should be explained carefully, and possibly discard all early revascularizations.

Response: We believe that our high rate of recurrent coronary revascularization procedures is in line with other studies in this distinct population. As stated in the original version, "Most recurrences (65\%) 
were new revascularization procedures, which is in line with other studies in distinct populations. For example, 58\% of recurrent events were coronary revascularization in the IMPROVE-IT trial in patients after an acute coronary syndrome [24]." To address this issue, we have now excluded any coronary revascularization recurrences that occurred within 30 days of the index event.

- It would be better to clarify precisely the reasons for revascularization.

Response: Unfortunately we did not collect this information, which is included as a limitation of the study (page 10). We have also excluded any coronary revascularization recurrences that occurred within 30 days of the index event.

-The mortality data are not of interest because they refer only to patients who have survived. While this information is prospective, only survivors can be studied, the rest of the information is retrospective.

Response: This is indeed another limitation of our retrospective study, but we believe that the number of deaths is interesting and we would prefer to retain it.

- Authors should define what they consider high intensity regime. Response: The definition for high-intensity regimens has been included in the manuscript. The definition is based on the percentage reduction of LDL-C concentration with a daily dose of statin: $>45 \%$ (i.e. atorvastatin 40 or $80 \mathrm{mg}$, rosuvastatin 20 or $40 \mathrm{mg}$ ) (Law MR, Wald NJ, Rudnicka AR. Quantifying effect of statins on low density lipoprotein cholesterol, ischaemic heart disease, and stroke: systematic review and meta-analysis. BMJ. 2003 Jun 28; 326: 1423).

We replaced the term "high intensity regimen" with the more obvious term "high-intensity statin".

-The units in the article contain many errors and are not homogeneous, sometimes, $\mathrm{mg} / \mathrm{dL}$, other $\mathrm{g} / \mathrm{L}$, and data are mixed.

Response: We sincerely apologize for the errors in the units for the lipid values, which have now been corrected and presented in $\mathrm{mg} / \mathrm{dL}$.

- Given that the time between the first event and the recurrence is very variable, the authors should present a table or char with the data per year, in this way it would be easier to calculate the post-event risk in the long term.

Response: We have included a Kaplan-Meier curve (figure 1), with the cumulative events per year. We have also added the data in Table 2.

\section{Figure 1: Kaplan-Meier curve of freedom from recurrent cardiovascular event.}

Coronary heart disease recurrences were censured when they were $<30$ days after a first coronary event. The shading shows $95 \%$ Hall-Wellner confidence interval. 


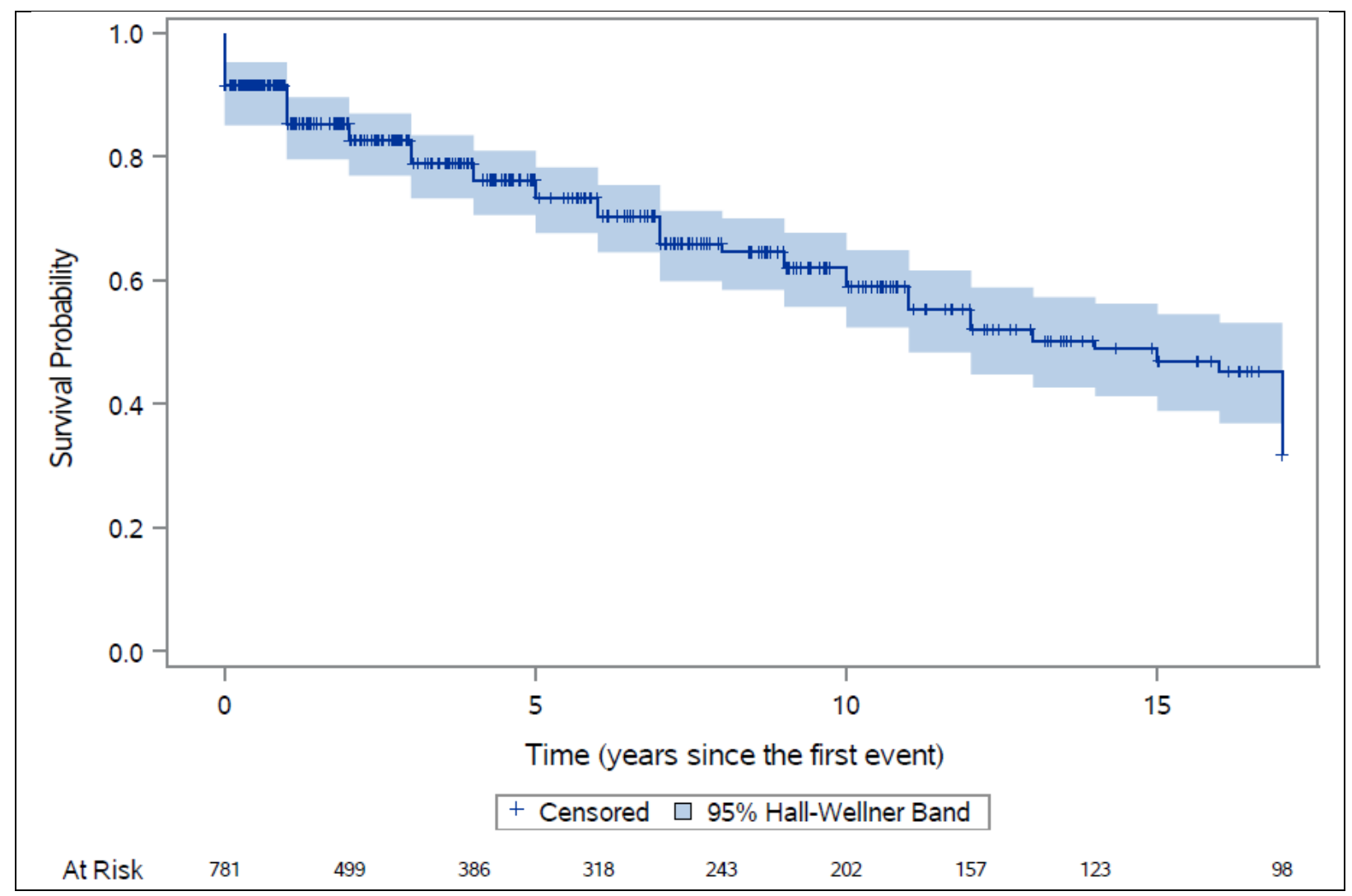

We could eventually also include another Kaplan-Meier curve, showing the freedom from recurrent cardiovascular events regarding the type of recurrent event (coronary revascularization, stroke, PAD, unstable angina and myocardial infarction). .

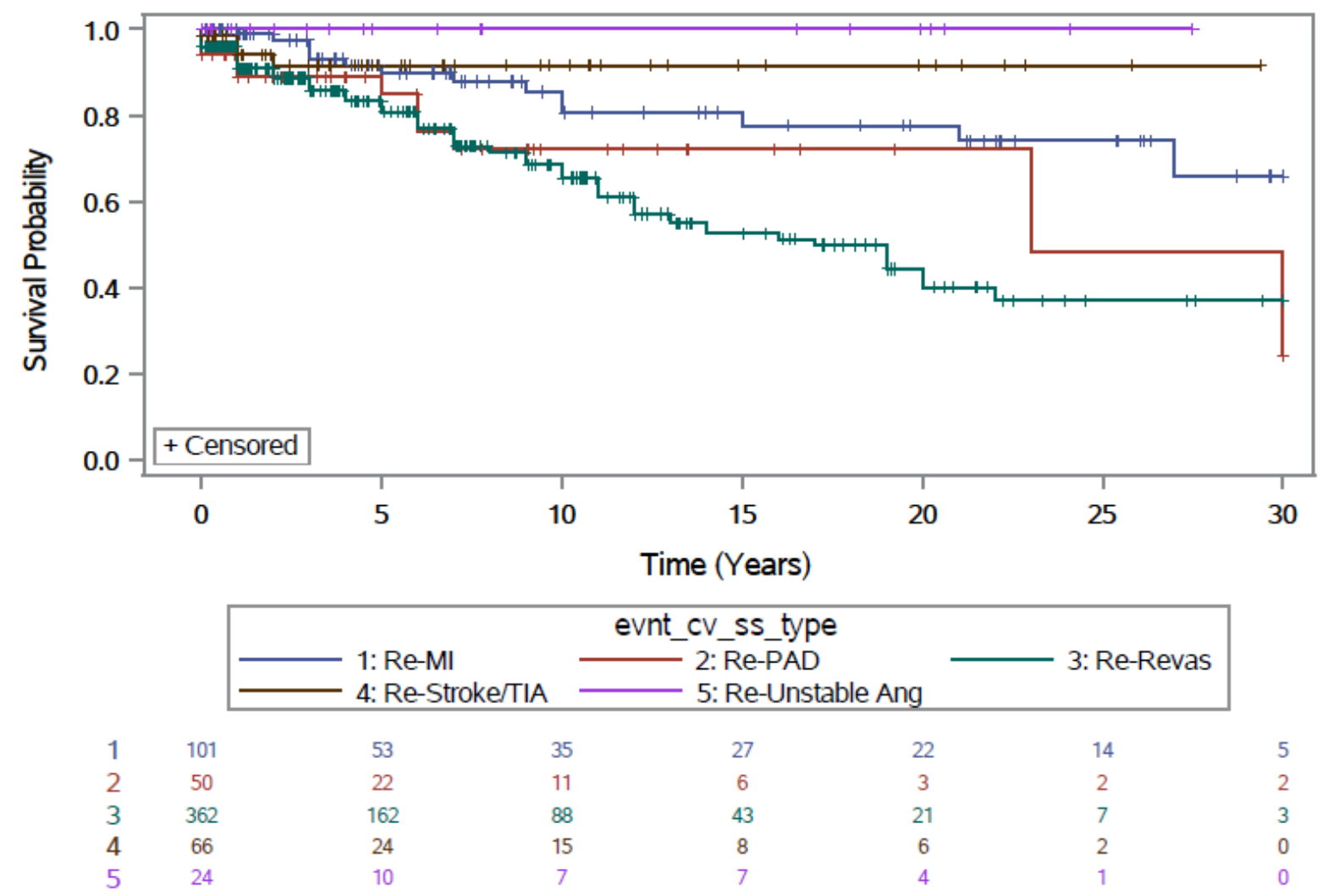


- A very important limitation is that information about medication is absent in half of the patients at the time of the event. This means that the data in Table 4 must be analyzed with caution.

Response: The reviewer has raised an important point: the absence of information on the lipidlowering therapy at the time of the event for nearly half of patients (45\%) is a limitation, and the data in the Table 4 must be interpreted with caution.

We have added the following sentence on pages 10-11: "Third, it is important to note that data regarding the initiation of statin therapy before the first event were absent for almost half of the patients, so the results on exposure on statins may not be representative of the entire population."

- In the discussion, the comparison of recurrences with other cohorts is very biased since the definition of recurrences is very different. In fact, without removing the events of the first year, the recurrences of major events: IAM and stroke is very low, less than expected in other populations. All the discussion and the title itself is somewhat biased magnifying the risk.

Response: We agree that the definitions for events and recurrent events vary between studies. We have commented on this crucial point (page 9 in the discussion): "The recurrence rates in these studies, albeit in different populations and with different endpoints, are markedly lower than in our registry of HeFH (9 per 100 patient-years)."

However, recurrences of $\mathrm{CV}$ events are frequent in the present cohort, even after the first year following the event. Indeed, $60 \%$ of $\mathrm{HeFH}$ patients had a CV recurrence $>1$ year later. We do not think that we are magnifying the risk. For example, in a Swedish registry, the prevalence of recurrent CV events in young patients ( $<60$ years; nevertheless older that the HeFh population of 46 years) was very low compared with our cohort: respectively 10\% vs 42\% (Jernberg, Eur Heart J 2015; 36:11631170).

We have now added a Kaplan-Meier curve (Figure 1) to clarify the point on the time of recurrences.

-There are multiple typographical errors in the quotes within the text, and typos that should be thoroughly reviewed.

Response: Thank you for raising this point. A native English speaker has reviewed the manuscript and corrected the language. 
Marseille, April $5^{\text {th }} 2018$,

\section{Dr A. von Eckardstein}

\section{Editor in Chief}

\section{Statement of originality:}

Dear A. von Eckardstein,

I certify that the data of the present study are my own work and have not been published in this or any substantially similar form, nor accepted, nor is it under consideration for publication elsewhere.

All authors have contributed to the preparation and development of this manuscript, and have approved the final draft prior to submission.

Thank you for considering the manuscript and I look forward to hearing from you.

Yours sincerely

Sophie BELIARD-LASSERRE, MD, PhD

APHM, Aix Marseille University, France

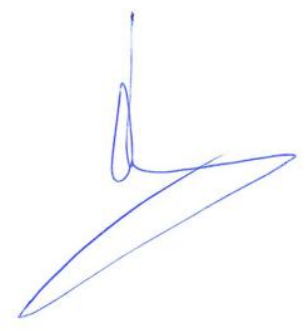




\section{REBUTTAL LETTER TO THE EDITOR AND THE REVIEWERS:}

Dear Professors von Eckardstein and Ray,

Thank you for reviewing our manuscript, entitled "High burden of recurrent cardiovascular events in heterozygous familial hypercholesterolemia: the French Familial Hypercholesterolemia Registry" (Ms. No. ATH-D-18-00445).

We again thank the reviewers for their overall positive comments and the pertinent points raised. Our responses to these comments are provided below, and the changes are marked in the manuscript.

We believe that the manuscript has benefited significantly from the reviewers' critiques, and hope that you will now find it acceptable for publication in your journal.

Yours sincerely,

Dr SOPHIE BELIARD, MD, PhD

Marseille, the 15 June 2018 ,

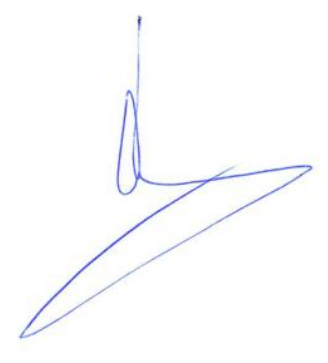


Marseille, April $5^{\text {th }} 2018$,

\section{Dr A. von Eckardstein}

\section{Editor in Chief}

Dear A. von Eckardstein,

This is the conflict of interest form for the present work:

SB, EB, FB, AC, JF, MF, MK, MV, SC, NP and JPR have received honoraria for boards, and/or conferences, and/or clinical trial and/or congress from Sanofi or Amgen.

AV has no conflict of interest to declare.

Thank you for considering the manuscript and I look forward to hearing from you.

Yours sincerely

Sophie BELIARD-LASSERRE, MD, PhD

APHM, Aix Marseille University, France

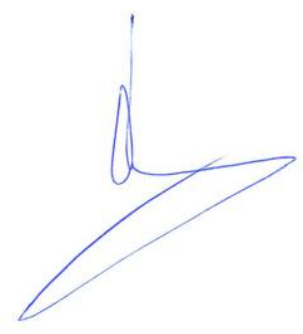


Figure 1: Kaplan-Meier curve of freedom from recurrent cardiovascular event.

Coronary heart disease recurrences were censured when they were $<30$ days after a first coronary event. The shading shows $95 \%$ Hall-Wellner confidence interval.

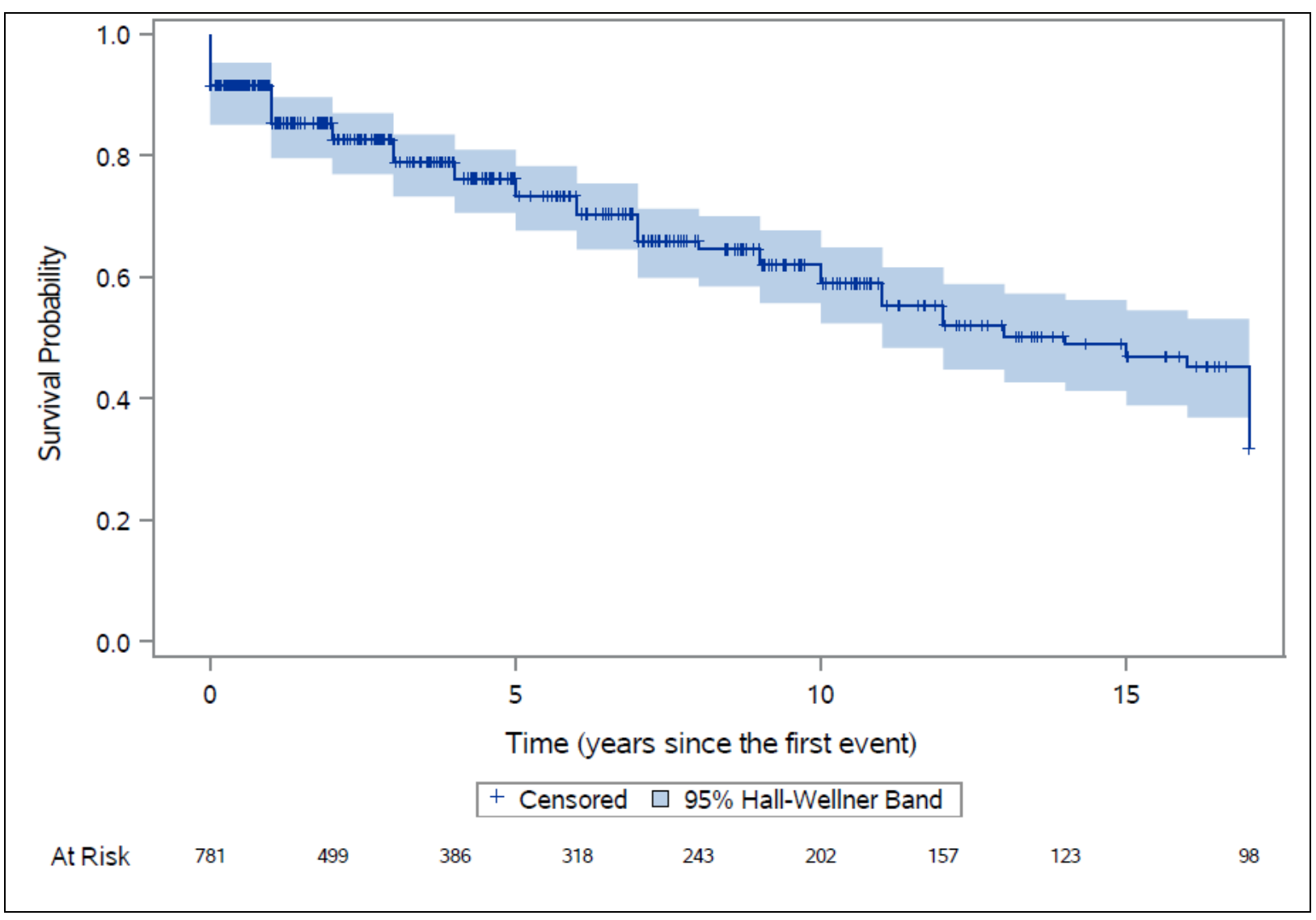


Table 1: Demographic and clinical characteristics of patients with heFH in secondary prevention (at the last visit to the lipid clinic).

\begin{tabular}{|lc|}
\hline Variables & Total (n=781) \\
\hline Men, $\mathrm{n}(\%)$ & $565(72)$ \\
\hline Age, years, mean (SD) & $60(13)$ \\
\hline Length of follow-up (from first cardiovascular event to last clinic visit), years, median (IQR) & $7(2-17)$ \\
\hline Patient-years of follow-up & 5779 \\
\hline Cardiovascular risk factors, $\mathbf{n}(\%)$ & $(\mathrm{n}=706)$ \\
$\quad$ Smoking status & $111(16)$ \\
$\quad$ Current & $277(39)$ \\
$\quad$ Former & $318(45)$ \\
$\quad$ Never smoker & $93(13)(\mathrm{n}=704)$ \\
Type 2 diabetes & $255(36)(\mathrm{n}=705)$ \\
Hypertension & $55(56)(\mathrm{n}=414)$ \\
Lipoprotein(a), mg/dL, mean (SD) & $501(64)(\mathrm{n}=781)$ \\
Familial history of CVD & $420(190)(\mathrm{n}=535)$ \\
Maximal total cholesterol, mg/dL, mean (SD) & $583(75)$ \\
\hline Patients who underwent genetic testing & $126(16)$ \\
Results not available & $20(3)$ \\
No mutation & $437(75)$ \\
Mutation ${ }^{a}$ & $401(92)$ \\
LDLR & $40(9)$ \\
APOB & $13(3)$ \\
\hline PCSK9 & \\
\hline APOB, apolpopros
\end{tabular}

APOB, apolipoprotein B; CT, maximum lifetime total cholesterol; heFH, heterozygous familial

hypercholesterolemia; IQR, interquartile range; LDLR, low-density lipoprotein receptor; SD, standard deviation.

${ }^{a}$ Some patients had more than one mutation. 
Table 2: Index and recurrent cardiovascular events (at the last visit to the lipid clinic).

\begin{tabular}{|c|c|}
\hline Variables & Total $(\mathrm{n}=781)$ \\
\hline Age at first event, years, median (IQR) & $47(39-55)(n=756)$ \\
\hline Type of first cardiovascular event, $\mathbf{n}(\%)$ & $(n=778)$ \\
\hline Coronary heart disease & $628(80)$ \\
\hline Coronary angioplasty or bypass & $403(64)$ \\
\hline Myocardial infarction & $167(27)$ \\
\hline Unstable angina & $50(6)$ \\
\hline Undefined & $11(1)$ \\
\hline Stroke or transient ischemic attack & $80(10)$ \\
\hline Peripheral artery disease ${ }^{a}$ & $68(9)$ \\
\hline Resuscitated sudden death & $2(<1)$ \\
\hline Type of subsequent events, $\mathrm{n}(\%)$ & $(n=511)$ \\
\hline Coronary heart disease & $400(78)$ \\
\hline Coronary angioplasty or bypass & $332(65)$ \\
\hline Myocardial infarction & $36(7)$ \\
\hline Unstable angina & $31(6)$ \\
\hline Undefined & $1(<1)$ \\
\hline Stroke or transient ischemic attack & $30(6)$ \\
\hline Peripheral artery disease ${ }^{a}$ & $76(15)$ \\
\hline Resuscitated sudden death & $2(<1)$ \\
\hline All-cause death, $\mathrm{n}(\%)$ & $18(2)$ \\
\hline Cardiovascular death, $\mathrm{n}(\%)$ & $8(1)$ \\
\hline \multicolumn{2}{|l|}{ Patients with recurrences, $\mathbf{n}(\%)$} \\
\hline$\geq 1^{\mathrm{D}}$ & $289(37)$ \\
\hline$\geq 2^{\mathrm{D}}$ & $111(14)$ \\
\hline$\geq 3^{\mathrm{D}}$ & $53(7)$ \\
\hline Recurrences per patient, mean (SD) & $1.8(1.4)$ \\
\hline Number of total recurrences & $n=511$ \\
\hline $\begin{array}{l}\text { Delay between initial event and first recurrence, months, } \\
\text { median (IQR) }\end{array}$ & $49(8-122)(n=281)$ \\
\hline$\leq 30$ days, $\mathrm{n}(\%)$ & $8(3)$ \\
\hline$>30$ days to <1 year, $\mathrm{n}(\%)$ & $81(29)$ \\
\hline $1-3$ years, $n(\%)$ & $37(13)$ \\
\hline$>3$ years, $n(\%)$ & $155(55)$ \\
\hline Delay between recurrences, months, median (IQR) & $38(9-104)(n=503)$ \\
\hline
\end{tabular}

IQR, interquartile range; SD, standard deviation.

${ }^{a}$ Carotid endarterectomy, carotid angioplasty, peripheral arterial bypass.

${ }^{\mathrm{b}}$ Recurrence of any cardiovascular event. 
Table 3 : Comparison of patients with and without a recurrent cardiovascular event.

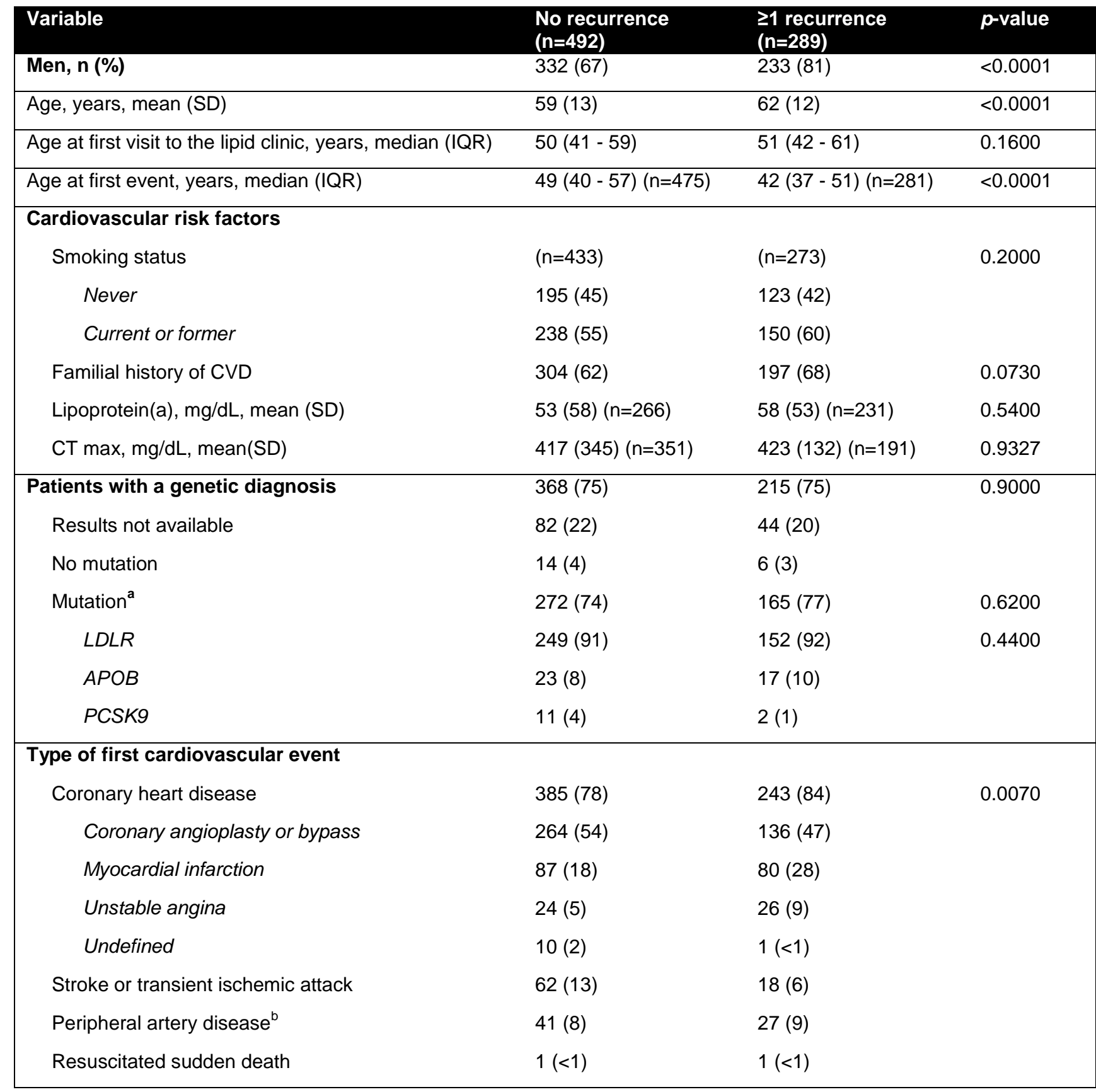

APOB, apolipoprotein B; CT, maximum lifetime total cholesterol; IQR, interquartile range; LDLR, lowdensity lipoprotein receptor; SD, standard deviation.

${ }^{\text {a }}$ Some patients had more than one mutation.

${ }^{\mathrm{b}}$ Carotid endarterectomy, carotid angioplasty, peripheral arterial bypass. 
Table 4: Treatments patterns in heFH patients and LDL-c levels, overall and with and without recurrent cardiovascular event, at the last clinic visit.

\begin{tabular}{|c|c|c|c|c|}
\hline Variable & $\begin{array}{l}\text { Total } \\
(n=781)\end{array}$ & $\begin{array}{l}\text { No } \\
\text { recurrence } \\
(n=492)\end{array}$ & $\begin{array}{l}\geq 1 \\
\text { recurrence } \\
(n=289)\end{array}$ & $p$-value \\
\hline History of statin exposition & $(n=430)$ & $(n=275)$ & $(n=155)$ & \\
\hline Age at start of statin treatment, years, median (IQR) & $41(33-50)$ & $41(32-50)$ & $41(34-49)$ & 0.7912 \\
\hline Overall exposure, years, median (IQR) & $19(10-27)$ & $16(8-26)$ & $23(15-28)$ & 0.0001 \\
\hline Exposure to statins before first event, $n(\%)$ & $208(48)$ & $147(53)$ & $61(39)$ & 0.0084 \\
\hline Duration of exposure to statins before first event, years & & & & 0.0191 \\
\hline Median (IQR) & $4(0-17)$ & $5(0-15)$ & $3(0-10)$ & \\
\hline Mean (SD) & $7.9(9.9)$ & $8.6(10.5)$ & $6.2(8.0)$ & \\
\hline \multicolumn{5}{|l|}{ Treatment at last visit } \\
\hline Statins (alone or in combination ) & $650(83)$ & $416(84)$ & $234(81)$ & 0.0690 \\
\hline High-potency statin $^{a}$ & $456(58)$ & $287(58)$ & $169(58)$ & \\
\hline Statin alone & $162(21)$ & $116(24)$ & $46(16)$ & \\
\hline Statins + ezetimibe & $448(57)$ & $281(57)$ & $167(58)$ & \\
\hline Statin + PCSK9 inhibitor & $15(2)$ & $6(1)$ & $9(3)$ & \\
\hline Statin + ezetimibe + PCSK9 inhibitor & $25(3)$ & $13(3)$ & $12(4)$ & \\
\hline Ezetimibe alone & $22(3)$ & $14(3)$ & $8(3)$ & \\
\hline PCSK9 inhibitor alone & $15(2)$ & $8(2)$ & $7(2)$ & \\
\hline Apheresis (alone or in combination) & $64(8)$ & $33(7)$ & $31(11)$ & \\
\hline Other treatment ${ }^{\mathrm{b}}$ & $23(3)$ & $17(3)$ & $6(2)$ & \\
\hline No treatment & $58(7)$ & $31(6)$ & $27(9)$ & \\
\hline LDL-c levels at last visit, mg/dL, mean (SD) & $(n=714)$ & $(\mathrm{n}=460)$ & $(n=254)$ & \\
\hline All patients & $144(75)$ & $148(78)$ & $136(68)$ & 0.0343 \\
\hline Patients on high-potency statin ${ }^{a}$ & $132(69)$ & $136(74)$ & $125(58)$ & 0.0725 \\
\hline Patients without treatment & $223(85)$ & $230(84)$ & $211(87)$ & 0.3627 \\
\hline
\end{tabular}

heFH, heterozygous familial hypercholesterolemia.

${ }^{\text {a }}$ Atorvastatin $40-80 \mathrm{mg}$, rosuvastatin $20-40 \mathrm{mg}$, simvastatin $>40 \mathrm{mg}$.

${ }^{\mathrm{b}}$ Fibrates, niacin, cholestyramine, yeast red rice, phytosterols. 


\section{${ }^{\star}$ Atherosclerosis style guide checklist}

I did it off line

I did it off line

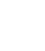

(2)

.

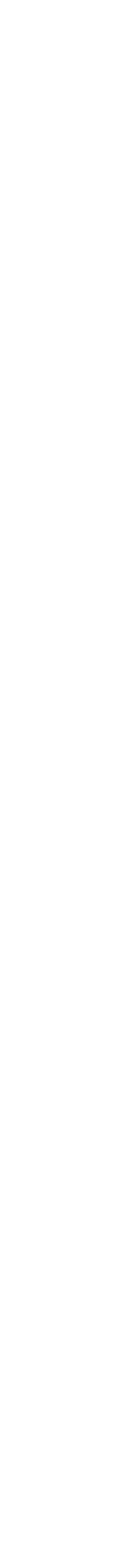

